\title{
Infant feeding practices in Soweto, South Africa: Implications for healthcare providers
}

\author{
S Nieuwoudt, ${ }^{1,2} \mathrm{MPH} ; \mathrm{L}$ Manderson,,${ }^{1,3} \mathrm{PhD} ; \mathbf{S}$ A Norris, ${ }^{2} \mathrm{PhD}$ \\ ${ }^{1}$ School of Public Health, Faculty of Health Sciences, University of the Witwatersrand, Johannesburg, South Africa \\ ${ }^{2}$ South African Medical Research Council Developmental Pathways Health and Research Unit, University of the Witwatersrand, Johannesburg, South Africa \\ ${ }^{3}$ Institute at Brown for Environment and Society, Brown University, Providence, RI, USA
}

Corresponding author: S Nieuwoudt (sara.nieuwoudt@wits.ac.za)

\begin{abstract}
Background. The 2011 Tshwane Declaration for the Promotion of Breastfeeding in South Africa ended the country's longstanding support for promoting either exclusive breastfeeding (EBF) or exclusive formula feeding for HIV-positive mothers. However, South Africa's EBF rate is only $32 \%$.

Objectives. To describe multilevel factors associated with different infant feeding practices among HIV-positive and negative mothers of infants aged $<6$ months in an HIV-endemic community.

Methods. A cross-sectional survey was administered to 298 HIV-positive and negative mothers accessing care in one of five community health clinics in Soweto, Johannesburg, between September 2015 and May 2016. Infant feeding practices and associated factors were explored through descriptive and multivariate analysis.

Results. Excluding HIV-positive mothers who chose formula feeding ( $n=97)$, breastfeeding initiation was almost universal (99.5\%). Caesarean section (CS) was the most common reason mothers delayed breastfeeding. HIV-positive mothers were significantly more likely to report prolonged EBF (and formula feeding) practices than their HIV-negative counterparts. Breastfeeding mothers were significantly more likely to be unemployed than mothers who formula fed. Mixed feeding was common.

Conclusions. EBF remains strongly associated with HIV status as opposed to infant health and development. Breastfeeding support for working mothers is needed. While breastfeeding increased following the Declaration, more should be done in the health setting to communicate the risks of mixed feeding in the first 6 months. The high rate of CSs reported by mothers, linked to late initiation of breastfeeding, also needs the medical community's attention.
\end{abstract}

S Afr Med J 2018;108(9):756-762. DOI:10.7196/SAMJ.2018.v108i9.13358

Breastfeeding is optimal for health and development over the life course, including for infants exposed to HIV. ${ }^{[1]}$ Exclusive breastfeeding (EBF) for the first 6 months is the best nutritional start for all infants, given the right conditions. ${ }^{[2,3]}$ However, mothers need to work around practical challenges and negotiate individual, familial, community (including health settings) and societal factors to select a feeding option (or feeding options) that best fits with their lives. ${ }^{[4,5]}$

The 2011 Tshwane Declaration for the Promotion of Breastfeeding in South Africa (hereafter, Declaration) ${ }^{[6]}$ is considered the turning point for breastfeeding in South Africa (SA). ${ }^{[7]}$ The Declaration altered SA's programme to eliminate mother-to-child transmission of HIV, firstly by dismantling the free formula programme in public facilities for HIV-positive mothers, and secondly by requiring healthcare workers to promote EBF only, rather than EBF or exclusive formula feeding (EFF). The Declaration therefore transformed a longstanding programme in the public healthcare system and shifted the balance of feeding options for HIV-positive mothers. SA's latest EBF estimate of $32 \%,{ }^{[8]}$ although below the $37 \%$ average for lowand middle-income countries, ${ }^{[1]}$ is well above the rates of $<10 \%$ documented between 1998 and 2011. ${ }^{[7,9,10]}$

We hypothesised that the higher EBF rates following the Declaration were associated with high early breastfeeding initiation practices and increased support from healthcare workers. We also believed that SA's HIV epidemic would influence feeding patterns according to HIV status. As conceptualised in Fig. 1, we also measured factors, including those within the healthcare setting, that the literature has shown to influence infant feeding practices, ${ }^{[5]}$ focusing on an HIV- endemic community where infant formula was previously provided. To test our hypothesis, we compared the profiles of HIV-positive and negative mothers, and explored detailed measures of infant feeding, stratified by infant age. Finally, we tested associations with current EBF through multivariate analysis.

\section{Methods}

We conducted a quantitative cross-sectional survey of 298 HIVpositive and negative mothers with infants $<6$ months of age who accessed community health clinics (CHCs) in Soweto, southwestern Johannesburg, between September 2015 and April 2016.

\section{Study setting}

The study was conducted in one health subdistrict of Soweto, serving a population of 1.3 million. Study participants were recruited from five $\mathrm{CHCs}$, where primary healthcare was provided free of charge; each averaged between 50 and 100 new pregnancy bookings per month. ${ }^{[11]}$ The CHCs from which we recruited had antenatal clinic HIV rates of $\sim 30 \%$, consistent with city-wide and national estimates of prevalence. ${ }^{[12]}$ The phase-out of infant formula had been completed before the end of 2012 .

\section{Ethical considerations}

The study protocol received ethics approval from the University of the Witwatersrand Human Research Ethics Committee (ref. no. M140803), the City of Johannesburg Research Committee (ref. no. 2014-15/068) and the five facilities. Participant screening, 
consent and data collection were managed by two female research assistants (RAs) who had previous research experience and were residents of Soweto. Both were trained in the protocol by the first author prior to data collection. The RAs' ability to speak a mixture of local languages, including isiZulu, Setswana and Sepedi, and their familiarity with the community enabled participants to seek clarification during the screening and consent processes as well as during data collection. All raw data were managed and stored securely by the first author.

\section{Population and sampling}

The study population for the questionnaire interviews comprised any mother of a singleton infant accessing public health facilities in the study area. Mothers had to be aged $\geq 18$ years with known HIV status, and the infants had to be aged $\leq 6$ months at the time of data collection, which took place at a different time to screening.

The study applied a quota sampling approach, seeking 50 mothers in each of six groups (Table 1). Our primary interest was in comparing feeding practices among HIV-positive mothers after the Declaration, segmented by infant ages: younger $(<12$ weeks) and older (12 - 36 weeks). We wanted sufficient HIV-positive mothers who were formula-feeding or breastfeeding to identify differentiating factors, and to compare them with HIV-negative mothers of infants in both age categories.

\section{Data collection}

The RAs screened eligible participants at the five facilities, approaching mothers in the postnatal, Integrated Management of Childhood Illness, Expanded Programme on Immunisation and labour wards. Of the 968 eligible mothers identified, 303 selfreported that they were HIV-positive. Of those, $51.0 \%$ reported that their primary feeding method was breastfeeding and the remaining $49.0 \%$ reported formula feeding. HIV-negative mothers were not asked their feeding method. After screening, only 862 mothers $(89.0 \%)$ could be reached telephonically for an appointment. Of these, 55 were ineligible because their infants were too old or they had moved. A further 36 refused. The RAs booked appointments with all other women according to availability and based on quota targets. Interviews continued until quota targets were reached. In all, 310 questionnaires were completed. Twelve were discarded because the infant was $>6$ months old, leaving 298 (Fig. 2).

The structured questionnaire consisted of five sections: sociodemographic infor-

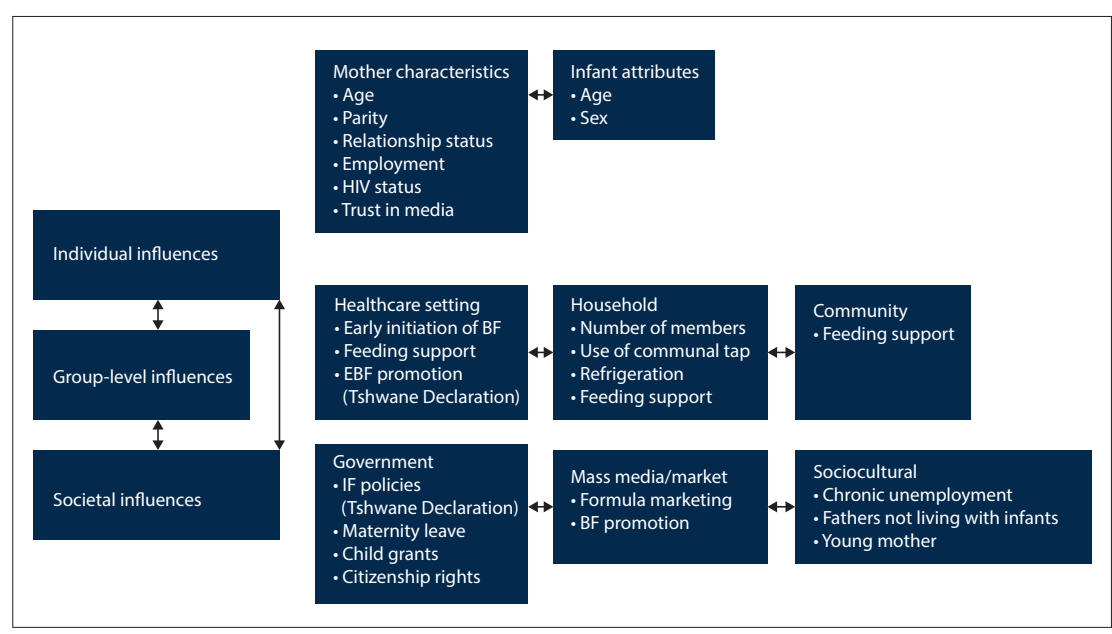

Fig. 1. Conceptual framework for infant feeding. $(B F=$ breastfeeding; $E B F=$ exclusive $B F ; I F=$ infant feeding.) mation, initiation of infant feeding, current infant feeding, current health status of mother and infant, and sources of infant feeding information. The two infant feeding sections comprised questions developed by the WHO, ${ }^{[13]}$ some of which were adapted to better reflect locally available food. Further amendments were made in the first week to correct skip patterns. These data were retained, as the RAs caught the mistakes. The RAs interviewed participants using paper-based questionnaires. Most interviews took place in a private room at the study office, with a minority conducted in mothers' homes. Interviews took on average 20 minutes to complete after the consent process.

\section{Data management and analysis}

The RAs captured and managed data on the REDCap (Research Electronic Data Capture) electronic data capture tool hosted at the University of the Witwatersrand. The first author monitored this for quality assurance. Discrepancies were checked against the original questionnaires. Data were uploaded into Stata version 13 (StataCorp, USA) for data cleaning, transformation of variables and analysis by the first author.

Feeding outcome measures were calculated both over the infant's lifetime and as a current feeding measure of 24-hour recall recommended by the WHO. ${ }^{[14]}$ Lifetime measures included ever breastfeeding, time to initiation and transformed variables of $\mathrm{EBF}$, predominant breastfeeding, EFF, and mixed feeding, using WHO definitions. ${ }^{[13]}$ Current feeding measures included EBF, predominant breastfeeding, EFF and mixed feeding over the past 24-hour period, using a detailed tool. Mothers were also asked about the main modality of feeding (breast, bottle, cup or spoon).

Guided by the conceptual framework, individual, group and social-level variables were considered. Individual variables for mothers included age, parity, self-reported HIV status, relationship status, employment and media habits, such as where the mother obtained information about breastfeeding,

Table 1. Number of mothers by study sample group

\begin{tabular}{llll}
\hline & \multicolumn{2}{c}{ Infant age at questionnaire (weeks) } & Total \\
\cline { 2 - 3 } Reported HIV status ${ }^{*}$ and feeding at screening & $<\mathbf{1 2}$ & $\mathbf{1 2 - 3 6}$ & 99 \\
\hline HIV+, breastfeeding, $n$ & 49 & 50 & 97 \\
HIV+, formula feeding, $n$ & 48 & 49 & 102 \\
HIV-, any feeding, $n$ & 53 & 49 & 298 \\
Total, $N$ & 150 & 148 & \\
HIV+ $=$ HIV-positive; HIV- $=$ HIV-negative. \\
*HVV-positive status reported in the questionnaire and 'main' modality reported during study screening. Five women reported being HIV- at time of screening and HIV+ at the time of \\
questionnaire adminitration.
\end{tabular}




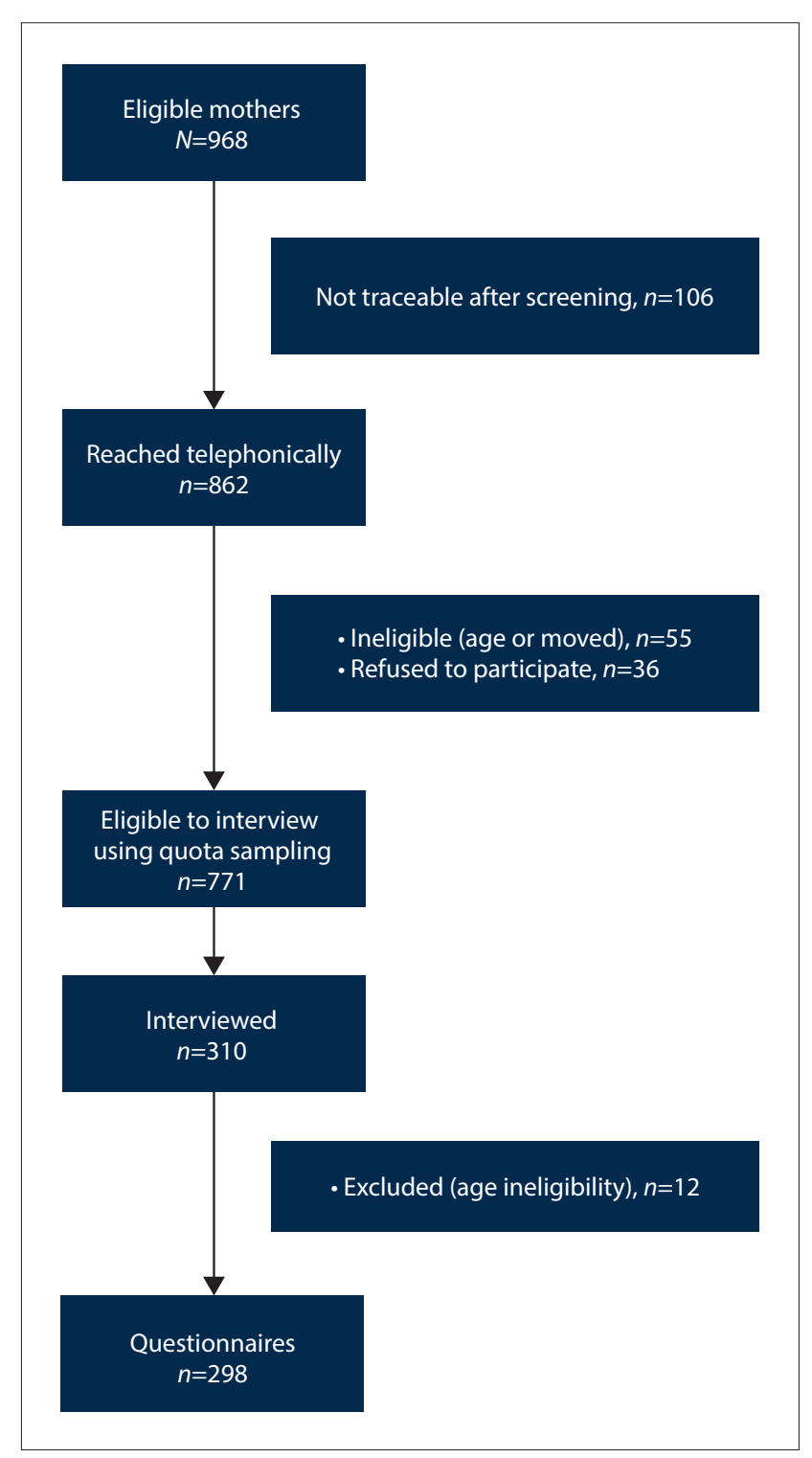

Fig. 2. Mother participation flow diagram.

mobile phone habits and most trusted sources of information. The infant's age and sex were also captured. Clinic-related indicators included early initiation of breastfeeding, reasons if not, and whether healthcare workers had supported mothers. For households, we captured the household size, availability of refrigeration, use of a communal tap, and support for breastfeeding. Community support for breastfeeding was also captured as a response item. Government social welfare was captured through the Child Support Grant programme. Nationality was recorded, as discriminatory policies and practices against migrants have been noted ${ }^{[15]}$ Participants also were asked about breastfeeding promotion in relation to media.

Descriptive analysis was conducted on all outcome, explanatory and confounding variables, followed by bivariate analysis to shortlist variables for inclusion in regression analysis, using $t$-test, $\chi^{2}$ and Wald test statistics, with a cut-off of $p<0.20$. To address potential confounding, we tested for collinearity prior to developing multivariate logistic models for EBF. The selection of variables for the models was based on bivariate analysis as well as theory. Key constructs supported by the literature were forced into the model, such as breastfeeding support and HIV status, even if they did not meet the cut-off. We used forward logistic regression to construct the models based on a theoretical approach and tested for interaction effects, resulting in three models.

\section{Results}

Mothers were recruited into the study based on their self-reported HIV status and feeding practices. A total of 298 mothers completed interviews for analysis (Table 1).

Table 2 presents mothers' sociodemographic characteristics and exposure to breastfeeding promotion. This was a population of mothers accessing CHCs who came from vulnerable backgrounds. Although nearly all owned a mobile phone, three-quarters were unemployed, and over half accessed child grants and relied on communal taps for drinking water. Of the unemployed, only 13 (4.4\%) were students. Most women lived in households averaging 4.5 individuals, yet only $40.3 \%$ lived with the father of their child. Most were South African. Markers of socioeconomic vulnerability persisted across groups. Antiretroviral (ARV) use among the HIVpositive population (not shown) was high, with $99.5 \%$ of those who responded $(n=191)$ reporting current ARV use. This did not differ by feeding practice.

Differences between HIV-positive and HIV-negative mothers were limited. HIV-positive mothers were significantly older than their HIV-negative counterparts, possibly owing to the sampling strategy, which required HIV disclosure. Consistent with their older age, they were also more likely to be multiparous $(82.7 \%)$ than those who were not infected (60.8\%). Finally, they were significantly more likely to rely on communal taps (62.2\%) than their HIV-negative counterparts (48.0\%).

Main feeding content was also a source of differentiation. Formulafeeding mothers were significantly more likely to be employed (35.6\%) than their breastfeeding counterparts (16.3\%). For HIVnegative mothers, those who were formula feeding were significantly less likely to access water from a communal tap. Aligned with their higher employment, the HIV-negative formula-feeding mother group was wealthier than the three other groups.

Table 2 also highlights whether women had ever received breastfeeding promotion messages on their mobile phone, whether they had any support to breastfeed $(N=228)$, and their most trusted source of breastfeeding information (if any; $N=253$ ). Of the 98 mothers who reported receiving information on their phones, $87.8 \%$ reported their source as MomConnect, which is sponsored by the National Department of Health. Healthcare workers and family also provided direct breastfeeding support and were named as trusted sources of information, with $81 \%$ of women citing either nurses or other hospital staff as their most important source of information.

Infant feeding practices were explored by infant age, with the exception of ever breastfeeding and breastfeeding initiation (Table 3). The proportion of infants ever breastfed was $76.5 \%$; this includes 28.9\% of HIV-positive women electing to formula feed who reported ever feeding their infants breastmilk. For mothers who breastfed, only $58.4 \%$ achieved early initiation. In a pooled analysis of breastfeeding initiation (not shown), $22.0 \%$ of those who delayed initiation reported that this was due to delivering via caesarean section (CS). The same proportion reported that their infants had been taken to an intensive care unit. Over $10 \%$ said they were not given their baby immediately, and another $10 \%$ reported that their infants were premature (it was unclear whether their infants had trouble latching or they were not given their infants). Another cause for delayed initiation was 'milk not coming in'. Other reasons were that either the mother or the infant was sleeping. 
Table 2. Mothers' characteristics, overall and by self-reported HIV status and feeding content

\begin{tabular}{|c|c|c|c|c|c|}
\hline \multirow[b]{2}{*}{ Mother/infant characteristics } & \multirow[b]{2}{*}{ Overall $(N=298)$} & \multicolumn{2}{|c|}{ HIV+ $(N=196)$} & \multicolumn{2}{|c|}{ HIV- $(N=102)$} \\
\hline & & $\mathrm{BF}(n=99)$ & FF $(n=97)$ & BF $(n=67)$ & FF $(n=35)$ \\
\hline Mother's age (years), median (IQR) & $29(25-33)$ & $30(25-35)^{*}$ & $31(27-34)^{*}$ & $27(23-31)$ & $26(21-32)$ \\
\hline Infant's age (days), median (IQR) & $89(44-118)$ & $90(45-115)$ & $90(38-126)$ & $71(38-112)$ & $71(38-112)$ \\
\hline Infant sex male, $n(\%)$ & $137(46.3)$ & $46(46.5)$ & $48(50.0)$ & $26(39.4)$ & $17(48.6)$ \\
\hline Household size, median (IQR) & $4.5(3-6)$ & $5(3-6)$ & $4(3-4)$ & $4(4-6)$ & $5(4-7)$ \\
\hline Multiparous, $n(\%)$ & $224(75.2)$ & $80(80.8)^{\star *}$ & $82(84.5)^{* *}$ & $44(65.7)$ & $18(51.4)$ \\
\hline South African, $n(\%)$ & $259(87.2)$ & $85(85.9)$ & $82(84.5)$ & $60(89.6)$ & $32(94.1)$ \\
\hline Unemployed, $n(\%)$ & $224(75.2)$ & $83(83.8)^{*}$ & $62(63.9)$ & $56(83.6)^{*}$ & $23(65.7)$ \\
\hline \multicolumn{6}{|l|}{ Relationship status, $n(\%)$} \\
\hline Single & $71(23.8)$ & $29(29.3)$ & $23(23.7)$ & $11(16.4)$ & $8(22.8)$ \\
\hline Not living with partner & $107(35.9)$ & $34(34.3)$ & $35(36.1)$ & $23(34.3)$ & $23(42.9)$ \\
\hline Living with partner & $120(40.3)$ & $36(36.4)$ & $39(40.2)$ & $33(49.3)$ & $12(34.3)$ \\
\hline Child grant, $n(\%)$ & $167(56.0)$ & $60(60.6)$ & $53(54.6)$ & $40(59.7)$ & $14(40.0)$ \\
\hline Uses communal tap, $n(\%)$ & $171(57.4)$ & $63(63.3)^{* *}$ & $59(60.8)$ & $40(59.7)^{* *}$ & $9(25.7)$ \\
\hline No refrigeration, $n(\%)$ & $38(12.8)$ & $13(13.3)$ & $17(17.5)$ & $7(10.5)$ & $1(2.9)$ \\
\hline Owns mobile phone, $n(\%)$ & $294(98.7)$ & $96(97.0)$ & $97(100)$ & $66(98.5)$ & $35(100)$ \\
\hline BF info on mobile, $n(\%)$ & $98(33.5)$ & $32(33.7)$ & $32(33.0)$ & $23(34.9)$ & $11(31.4)$ \\
\hline Main BF supporter $(N=228)^{\dagger}, n(\%)$ & $180(79.0)$ & $83(83.8)$ & $21(75.0)$ & $50(74.6)$ & $26(76.5)$ \\
\hline Partner & $21(11.7)$ & $15(18.1)$ & $1(4.8)$ & $4(8.0)$ & $1(3.9)$ \\
\hline Family/friends & $55(30.6)$ & $20(24.1)$ & $5(23.8)$ & $17(34.0)$ & $13(50.0)$ \\
\hline Healthcare worker & $104(57.8)$ & $48(57.8)$ & $15(71.4)$ & $29(58.0)$ & $12(46.2)$ \\
\hline Trusted BF source $(N=253)^{\ddagger}, n(\%)$ & $253(85.6)$ & $86(86.9)$ & $87(89.7)$ & $52(77.6)$ & $28(80.0)$ \\
\hline Clinic/hospital staff & $205(81.0)$ & $73(84.9)$ & $74(85.1)$ & $39(75.0)$ & $19(67.9)$ \\
\hline Family/friends & $14(5.5)$ & $2(2.3)$ & $4(4.6)$ & $4(7.7)$ & $5(17.9)$ \\
\hline Mass media & $34(13.4)$ & $11(12.8)$ & $9(10.3)$ & $9(17.3)$ & $4(14.3)$ \\
\hline
\end{tabular}

Mothers were asked what their infant had ingested during her or his lifetime as a multiple-choice question early in their interview. The rate of lifetime EBF was $44.0 \%$ for younger infants and $31.8 \%$ for older infants. For EFF the proportion was also higher for younger infants. Mixed feeding, conversely, increased with infant age, with a corresponding increase in bottle use for older infants. Unlike current feeding, the lifetime mixed-feeding measure was unable to show whether breastmilk and formula were given concurrently or mothers had shifted from one to the other during the infant's life. The rate of predominant breastfeeding was relatively low regardless of infant age. Highly significant differences by HIV status and feeding type followed expected trends given the sampling strategy employed.

Current feeding practices, based on a 24-hour recall, allowed us to measure mixed feeding (Table 3). Current EBF and EFF rates were higher than the lifetime measures, again with mixed feeding increasing with infant age. The rate of exclusive feeding, especially EFF but also EBF, was greater among the HIV-positive mothers.

Bivariate analysis was conducted for both 24-hour and lifetime measures of $\mathrm{EBF}$ to determine which variables to include in regression models, using a cut-off of $p<0.20$. The infant's age, unemployment, accessing a child grant, mobile phone ownership, main breastfeeding supporter and most trusted source of breastfeeding information were significant. Early initiation of breastfeeding and parity met the significance cut-offs for lifetime EBF only, but were retained in the model for theoretical reasons. HIV status, relationship status and the presence of breastfeeding support were also retained for theoretical reasons.

All logistic regression models were adjusted for infant age. Model 1 included individual sociodemographic variables, including HIV status $(N=298)$. Unemployed mothers had nearly double the odds (1.88, 95\% CI $1.03-3.44)$ of exclusively breastfeeding compared with those who were employed. No other individual-level factors were significant in model 1 (Table 4).

HIV status in the first model was insignificant, but it was highly significant in the subsequent models. Mothers who had never breastfed were excluded from model 2, reducing the sample size $(N=228)$. When the behaviour of early initiation of breastfeeding was added to the logistic regression (model 2), employment became insignificant, and EBF was associated with being HIV-positive and receiving a child grant. The model's explanation of variance more than doubled to 0.118 . In model 3 , the participants' main breastfeeding support was added. In this final model, HIV status was the only factor that was retained as significant. There was no interaction between HIV status and early initiation of breastfeeding.

\section{Discussion}

This study reconfirms the trend of EBF increasing in Soweto. The measures of $44.0 \% \mathrm{EBF}$ for infants aged $<3$ months and $31.8 \%$ for infants aged $3-6$ months are in keeping with the national statistics of $32.0 \%{ }^{[8]}$ though lower that the EBF rate of $68.0 \%$ in the Soweto study. ${ }^{[10]}$ The reason for the other study's higher EBF rate could be the sampling method (convenience v. quota), sample size ( $N=100 \mathrm{v}$. $N=298$ ), or how infant feeding was measured. We used the recommended WHO EBF measure. ${ }^{[13,14]}$ The sociodemographic characteristics of the two study populations were similar. Both studies reported high ARV use among HIV-positive mothers in Soweto: $99.5 \%$ for this study and $87.9 \%$ of postpartum mothers in the previous study. ${ }^{[10]}$ This is in line with the 92.1\% ART initiation rate reported by the government for Gauteng 
Table 3. Infant feeding, overall, by self-reported HIV status and by feeding practice, segmented by infant age

\begin{tabular}{|c|c|c|c|c|c|}
\hline \multirow[b]{2}{*}{ Infant feeding practices } & \multirow[b]{2}{*}{ Overall $(N=298), n(\%)$} & \multicolumn{2}{|c|}{$\mathrm{HIV}+(N=97)$} & \multicolumn{2}{|c|}{ HIV- $(N=53)$} \\
\hline & & BF $(n=99)$ & FF $(n=97)$ & $\mathrm{BF}(n=67)$ & FF $(n=35)$ \\
\hline \multicolumn{6}{|l|}{ Lifetime feeding } \\
\hline Ever breastfed & $228(76.5)$ & $99(100)$ & $28(28.9)$ & $67(100)$ & $34(97.1)$ \\
\hline Early initiation & $125(54.8)$ & $56(56.6)$ & $16(57.1)$ & $41(61.2)$ & $12(35.3)$ \\
\hline$<3$ months & $150(50.3)$ & $49(49.5)$ & $48(49.5)$ & $39(58.2)$ & $14(40.0)$ \\
\hline \multicolumn{6}{|l|}{ Lifetime feeding } \\
\hline Exclusively breastfed & $66(44.0)$ & $31(83.7)$ & 0 & $25(64.1)$ & \\
\hline Pred. breastmilk & $11(7.3)$ & $5(10.2)$ & 0 & $6(15.4)$ & 0 \\
\hline Mixed feeding* & $48(32.0)$ & $3(6.1)$ & $23(47.9)$ & $8(20.5)$ & $14(100)$ \\
\hline Exclusively formula & $25(16.7)$ & 0 & $25(52.1)$ & 0 & 0 \\
\hline \multicolumn{6}{|l|}{ Current feeding } \\
\hline Exclusively breastfed & $69(46.0)$ & $42(85.7)$ & 0 & $27(69.2)$ & 0 \\
\hline Pred. breastmilk & $6(4.0)$ & $3(6.1)$ & 0 & $3(7.7)$ & 0 \\
\hline Mixed feeding & $37(24.7)$ & $4(8.2)$ & $16(33.3)$ & $9(23.1)$ & $8(57.1)$ \\
\hline Exclusively formula & $38(25.3)$ & 0 & $32(66.7)$ & 0 & $6(42.9)$ \\
\hline Mainly formula & $63(42.0)$ & $1(2.0)$ & $48(100)$ & $1(2.6)$ & $13(92.9)$ \\
\hline $3-6$ months & $148(49.7)$ & $50(50.5)$ & $49(50.5)$ & $28(41.8)$ & $21(60.0)$ \\
\hline \multicolumn{6}{|l|}{ Lifetime feeding } \\
\hline Exclusively breastfed & $47(31.8)$ & $34(68.0)$ & 0 & $13(46.4)$ & 0 \\
\hline Pred. breastmilk & $5(3.4)$ & $2(4.0)$ & 0 & $3(10.7)$ & 0 \\
\hline Mixed feeding ${ }^{*}$ & $77(52.0)$ & $14(28.0)$ & $30(61.2)$ & $12(42.9)$ & $21(100)$ \\
\hline Exclusively formula & $19(12.8)$ & 0 & $19(38.8)$ & 0 & 0 \\
\hline \multicolumn{6}{|l|}{ Current feeding } \\
\hline Exclusively breastmilk & $49(33.1)$ & $37(74.0)$ & 0 & $12(42.9)$ & 0 \\
\hline Pred. breastmilk & $6(4.1)$ & $1(2.0)$ & 0 & $5(17.9)$ & 0 \\
\hline Mixed feeding & 65 (43.9) & $12(24.0)$ & $22(44.9)$ & $11(39.3)$ & $20(95.2)$ \\
\hline Exclusively formula & $28(18.9)$ & 0 & $27(55.1)$ & 0 & $1(4.8)$ \\
\hline Mainly formula & $78(52.7)$ & $6(12.0)$ & $49(100)$ & $2(7.1)$ & $21(100)$ \\
\hline
\end{tabular}

Table 4. Factors associated with exclusive breastfeeding in past 24 hours, all models adjusted for infant age

\begin{tabular}{llll}
\hline Variables & $\begin{array}{l}\text { Individual, model 1 } \\
(\mathbf{N = 2 9 8}), \mathbf{O R}(\mathbf{9 5} \% \mathbf{C I})\end{array}$ & $\begin{array}{l}\text { Behaviour, model 2 } \\
(\mathbf{N = 2 2 8}), \mathbf{O R}(\mathbf{9 5} \% \mathbf{C I})\end{array}$ & $\begin{array}{l}\text { Support, model 3 } \\
(\mathbf{N = 1 8 0}), \mathbf{O R}(\mathbf{9 5} \% \mathrm{CI})\end{array}$ \\
\hline Unemployed & $1.88(1.03-3.44)^{*}$ & $1.52(0.73-3.16)$ & $1.08(0.45-2.56)$ \\
Receiving child grant & $1.55(0.91-2.61)$ & $2.04(1.09-3.80)^{*}$ & $1.88(0.92-3.86)$ \\
Multiparous & $1.29(0.71-2.36)$ & $1.75(0.90-3.42)$ & $1.78(0.81-3.91)$ \\
Relationship & & & - \\
$\quad-$ & - & $0.65(0.27-1.59)$ \\
$\quad$ Single & $0.82(0.43-1.55)$ & $0.75(0.35-1.60)$ & $0.94(0.36-2.41)$ \\
$\quad$ Living together & $0.74(0.40-1.39)$ & $0.81(0.38-1.75)$ & $1.02(0.50-2.08)$ \\
$\quad$ Not living together & $0.81(0.49-1.35)$ & $0.83(0.45-1.52)$ & $2.69(1.31-5.51)^{*}$ \\
HIV-positive & $0.99(0.58-1.68)$ & $2.45(1.36-4.42)^{*}$ & $1.14(0.57-2.28)$ \\
Immediately breastfed & - & $1.12(0.62-2.01)$ & - \\
Main breastfeeding support & & & $1.34(0.37-4.83)$ \\
$\quad$ Partner (Yes) & - & - & $2.42(0.80-7.36)$ \\
$\quad$ Health worker (Yes) & - & - & 0.135 \\
$\quad$ Family/friend (Yes) & - & 0.118 & \\
Model $R^{2}$ & 0.046 & & \\
OR=odds ratio; CI = confidence interval. & & &
\end{tabular}

Province ${ }^{[16]}$ and bodes well for reduced HIV transmission, even with slight lapses in exclusive feeding. ${ }^{[3]}$

Other SA studies have also observed that HIV-positive mothers have higher odds of maintaining EBF than their HIV-negative counterparts, ${ }^{[11,17]}$ probably because of fears of HIV transmission. ${ }^{[1,117]}$ Exclusive feeding practices among HIV-positive mothers have been attributed to intensive prevention of mother-to-child transmission counselling over the past decade, ${ }^{[17]}$ but with the result that in 
some cases exclusive feeding is perceived as an (involuntary) HIV disclosure. ${ }^{[18,19]} \mathrm{EBF}$ needs to be reframed as a child development issue. While the fear of transmission is an important factor that needs to be addressed in counselling HIV-positive mothers ${ }^{[20]}$ researchers have already shown that fear is insufficient to maintain breastfeeding exclusivity. ${ }^{[2]}$ Furthermore, $>70.0 \%$ of the mothers accessing clinics are not HIV-positive. Factors such as having supportive family environments to adopt clinical advice need much more attention ${ }^{[22]}$ for overall EBF promotion.

Mixed feeding increased in all groups as infants grew older, as one would expect with pressures for mothers to return to work and older infants beginning to show interest in food. Predominant feeding was lower than we expected. Despite evidence of the benefits of EBF for all infants, ${ }^{[1]}$ this has not been matched by messaging around the risks of mixed feeding beyond HIV transmission. The pressures mothers face to mixed-feed in SA are well documented and multifaceted, ${ }^{[7,20]}$ and a clearer communication strategy, particularly for HIV-negative mothers, is needed to persuade them of the benefits of EBF and/or the risks of mixed feeding for their infants.

The importance of the healthcare system in promoting infant feeding practices was highlighted in this study. Consistent with other SA studies, ${ }^{[7,23]}$ healthcare workers were trusted sources of support and key information sources. In addition, the health system connected mothers to mobile information through MomConnect, ${ }^{[24]}$ and many women gained information from this source. Although mobile messages were not reported as mothers' first source of information, they still play a reinforcing role.

This study also highlighted how efforts to reduce CSs may have a positive impact on EBF. Specifically, breastfeeding initiation is a critical period for the establishment of breastfeeding. ${ }^{[25]}$ Delayed initiation reduces the likelihood of EBF, and it is also associated with higher rates of neonatal mortality. ${ }^{[26]}$ As such, it is highly concerning that $>40.0 \%$ of mothers in this study initiated breastfeeding after $>1$ hour. Of these women, nearly a quarter attributed this to a CS. These rates seem realistic, with another study reporting a $21.5 \%$ CS rate in public facilities; ${ }^{[25]}$ for mothers covered by private medical schemes the rate is over 70\%. ${ }^{[27]}$ The WHO target for CSs is $<15 \%$, ${ }^{[25]}$ which would make a focus on CSs in line with global standards. To further support an argument to focus on CSs and breastfeeding initiation, the inclusion of early initiation into our regression model altered our study findings, suggesting that this is a key behavioural factor to support EBF.

We took care during interviews to make participants comfortable to speak freely. Nevertheless, the risk of social desirability influencing how mothers answered due to the place of recruitment, identity of interviewers or administration of the interview cannot be discounted. Infant feeding was self-reported. While this is the standard for most infant feeding studies, including those we compare our results with, discrepancies between self-reported and biologically confirmed breastfeeding have been noted. ${ }^{[28]}$ We addressed potential confounding by testing for collinearity and controlling for confounders and interactions in regression models.

The use of quota sampling may have introduced an element of sampling bias, although the RAs varied the times and days they visited clinics and circulated between all the wards to reduce other forms of selection bias. We intentionally oversampled HIV-positive mothers given our interest in the policy's impact on this population in terms of feeding practices. All descriptive tables were disaggregated to highlight these nuances; disaggregation also supports tailored interventions. Quota sampling reflected community practices and most sociodemographic characteristics of our sample were shared across groups. Fewer than $4.0 \%$ of eligible mothers refused to participate when contacted to book an appointment. We have presented our conclusions to reflect our use of non-parametric sampling, which limits our ability to generalise findings.

\section{Conclusions}

Despite breastfeeding increases, neither HIV-positive nor HIVnegative women have fully embraced EBF, to the detriment of infants and mothers. As a nation that has struggled to meet infant mortality and morbidity targets, SA cannot afford to ignore the arguments to

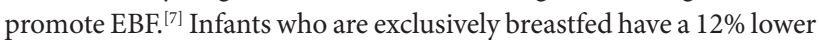
risk of dying than those who have not breastfed. ${ }^{[29]}$ Beyond increased mortality, EBF also confers a number of health and development benefits, such as reducing diarrhoeal and respiratory illnesses in the short term and higher intelligence in the longer term. ${ }^{[1]} \mathrm{EBF}$ is also associated with reduced breast cancer risk for mothers. ${ }^{[1]}$

To support EBF, we recommend that communication place greater emphasis on the developmental and health benefits of $\mathrm{EBF}^{[1]}$ and the risks of mixed feeding for all. In particular, these messages should be emphasised to nurses and frontline health practitioners through training, given how much mothers value them, and supplemented by communication from health providers to mothers, such as MomConnect. Communities also need to be engaged with these messages through mass media. Mothers also need other sectors' support and commitment, e.g. labour, for more supportive breastfeeding environments. In conclusion, we recommend multilevel responses that focus on improving the health setting, community and workplace environments to improve EBF uptake in SA to optimise infant growth and development. ${ }^{[4,5]}$

Acknowledgements. We acknowledge the contribution of the research co-ordinator/assistant Molebogeng Motlhatlhedi and assistant Deborah Makobe for their support in recruitment, consent, data collection and data capture. We thank Dr Juliana Kagura for support with assisting with early exploration of the data. We would also like to thank the Consortium for Advanced Research Training in Africa (CARTA) for sponsoring the first author to attend a seminar in Nigeria to finalise analysis in write-up, with access to senior statisticians and researchers.

Author contributions. SN was principal investigator in this study. She contributed to the conceptualisation of the study, writing of the study protocol and questionnaire, management of data collection, data cleaning, analysis and writing of the manuscript. LM contributed to the conceptualisation of the study and provided substantial inputs to the protocol and the writing of the manuscript. SAN is the principal investigator of the research programme in which this study was embedded. He contributed to the conceptualisation of the study, and provided substantial inputs to the protocol, data analysis and the writing of the manuscript.

Funding. The World Health Organization, Geneva, funded data collection for this study. The research was also supported by CARTA, which funds $\mathrm{SN}$ as a fellow. CARTA is jointly led by the African Population and Health Research Center and the University of the Witwatersrand and funded by the Carnegie Corporation of New York (grant no. B 8606.R02), Sida (grant no. 54100029) and the DELTAS Africa Initiative (grant no. 107768/Z/15/Z). The DELTAS Africa Initiative is an independent funding scheme of the African Academy of Sciences' Alliance for Accelerating Excellence in Science in Africa and supported by the New Partnership for Africa's Development Planning and Coordinating Agency (NEPAD Agency) with 
funding from the Wellcome Trust (UK) (grant no. 107768/Z/15/Z) and the UK government. The statements made and views expressed are solely the responsibility of the fellow, not the donor agencies.

Conflicts of interest. None.

1. Victora CG, Bahl R, Barros AJ, et al. Breastfeeding in the 21st century: Epidemiology, mechanisms, and lifelong effect. Lancet 2016;387(10017):475-490. https://doi.org/10.1016/S0140-6736(15)010242. World Health Organization. Infant and Young Child Feeding: Model Chapter for Textbooks for Medical Students and Allied Health Professionals. Geneva: WHO, 2009. http://www.who.int/ nutrition/publications/infantfeeding/9789241597494/en/ (accessed 10 April 2018).

3. World Health Organization, United Nations Children's Fund. Guideline: Updates on HIV and Infant Feeding: The Duration of Breastfeeding, and Support from Health Services to Improve Feeding Practices Among Mothers Living with HIV. Geneva: WHO, 2016. https://www.ncbi.nlm.nih.gov/ books/NBK379872/ (accessed 10 April 2018).

4. Lazarus R, Struthers H, Violari A. Promoting safe infant feeding practices - the importance of structural, social and contextual factors in southern Africa. J Int AIDS Soc 2013;16(1):18037. https:// doi.org/10.7448/IAS.16.1.18037

5. Rollins NC, Bhandari N, Hajeebhoy N, et al. Why invest, and what it will take to improve breastfeeding 5ollins NC, Bhandari N, Hajeebhoy N, et al. Why invest, and what it will take to improve breas
practices? Lancet 2016;387(10017):491-504. https://doi.org/10.1016/S0140-6736(15)01044-2

6. News. The Tshwane Declaration of Support for Breastfeeding in South Africa. S Afr J Clin Nutr 2011;24(4):214. http://www.sajcn.co.za/index.php/SAJCN/article/viewFile/586/820 (accessed 20 April 2018).

. Du Plessis L, Peer N, Honikman S, English R. Breastfeeding in South Africa: Are we making progress? In: Padarath A, King JF, Mackie E, Casciola J, eds. South African Health Review. Durban: Health Systems Trust, 2016:109-123.

8. National Department of Health, South Africa, Statistics South Africa, South African Medical Research Council, DHS Program, ICF. South Africa Demographic and Health Survey 2016: Key Indicators. Pretoria, South Africa, and Rockville, Md, USA: NDoH, Stats SA, SAMRC and ICF, 2017. https://www. statssa.gov.za/publications/Report\%2003-00-09/Report\%2003-00-092016.pdf (accessed 20 April 2018).

9. Tylleskär T, Jackson D, Meda N, et al. Exclusive breastfeeding promotion by peer counsellors in subSaharan Africa (PROMISE-EBF): A cluster-randomised trial. Lancet 2011;378(9789):420-427. https:// doi.org/10.1016/S0140-6736(11)60738-1

10. Mhlanga R. Maternal, newborn and child health: 30 years on. In: Barron P, Roma-Reardon J, eds. South African Health Review 2008. Durban: Health Systems Trust, 2008:115-128.

11. Mnyani CN, Tait CL, Armstrong J, et al. Infant feeding knowledge, perceptions and practices among women with and without HIV in Johannesburg, South Africa: A survey in healthcare facilities. Int Breastfeed J 2016;12:17. https://doi.org/10.1186/s13006-017-0109-x

12. National Department of Health, South Africa. The 2012 National Antenatal Sentinel HIV and Herpes Simplex Type-2 Prevalence Survey. 2014. https://www.health-e.org.za/wp-content/uploads/2014/05/ ASHIVHerp_Report2014_22May2014.pdf (accessed 20 April 2018).

13. World Health Organization, UNICEF, USAID, AED, UC-DAVIS, IFPRI. Indicators for Assessing Infant and Young Child Feeding Practices Part 2: Measurement. Geneva: WHO, 2010. http://www. who.int/nutrition/publications/infantfeeding/9789241599290/en/ (accessed 10 April 2018).

14. Abdel-Hady DM, El-Gilany AH. Calculating exclusive breastfeeding rates: Comparing dietary '24hour recall' with recall 'since birth' methods. Breastfeed Med 2016:11(10):514-518. https://doi. org $/ 10.1089 / \mathrm{bfm} .2016 .0032$
15. Makandwa T, Vearey J, eds. Giving birth in a foreign land: Exploring the maternal healthcare experiences of Zimbabwean migrant women living in Johannesburg, South Africa. Urban Forum 2017;28(1):75-90. https://doi.org/10.1007/s12132-017-9304-5

16. Massyn N, Peer N, English R, Padarath A, Barron P, Day C, eds. District Health Barometer 2015/16. Durban: Health Systems Trust, 2016

17. Jama NA, Wilford A, Masango Z, et al. Enablers and barriers to success among mothers planning to exclusively breastfeed for six months: A qualitative prospective cohort study in KwaZulu-Natal, South Africa. Int Breastfeed J 2017;12:43. https://doi.org/10.1186/s13006-017-0135-8

18. Ramara NS, Maputle MS, Lekhuleni ME. Infant feeding and HIV positive mothers in the Capricorn District of Limpopo province. Curationis 2010;33(1):5-16

19. Madiba S, Letsoalo R. HIV disclosure to partners and family among women enrolled in prevention of mother to child transmission of HIV program: Implications for infant feeding in poor resourced communities in South Africa. Glob J Health Sci 2013;5(4):13. https://doi.org/10.5539/gihs.v5n4p1

20. Tuthill E, McGrath J, Young S. Commonalities and differences in infant feeding attitudes an practices in the context of HIV in sub-Saharan Africa: A metasynthesis. AIDS Care 2014;26(2):214225. https://doi.org/10.1080/09540121.2013.813625

21. Doherty T, Chopra M, Nkonki L, Jackson D, Persson L-A. A longitudinal qualitative study of infant-feeding decision making and practices among HIV-positive women in South Africa. J Nutr 2006; 136(9):2421-2426. https://doi.org/10.1093/jn/136.9.2421

22. Mphego Z, Madiba S, Ntuli B. The influence of the family on adherence to exclusive breastfeeding: Experiences of women living in extended family households in poorly resourced communities of Mpumalanga Province, South Africa: Child nutrition and feeding practices. Afr J Phys Health Educ Recreat Dance 2014;20(Suppl 1):279-290. http://hdl.handle.net/10520/EJC162271 (accessed 3 August 2018).

23. Sibeko L, Coutsoudis A, Nzuza S, Gray-Donald K. Mothers' infant feeding experiences: Constraint and supports for optimal feeding in an HIV-impacted urban community in South Africa. Public Health Nutr 2009;12(11):1983-1990. https://doi.org/10.1017/S1368980009005199

24. Barron P, Pillay Y, Fernandes A, Sebidi J, Allen R. The MomConnect mHealth initiative in South Africa: Early impact on the supply side of MCH services. J Public Health Policy 2016;37(Suppl 2):201212. https://doi.org/10.1057/s41271-016-0015-2

25. Gebhardt GS, Fawcus S, Moodley J, Farina Z. Maternal death and caesarean section in South Africa: Results from the 2011 - 2013 Saving Mothers Report of the National Committee for Confidential Enquiries into Maternal Deaths. S Afr Med J 2015;105(4):287-291. https://doi.org/10.7196/ SAMJ.9351

26. Edmond KM, Zandoh C, Quigley MA, Amenga-Etego S, Owusu-Agyei S, Kirkwood BR. Delayed breastfeeding initiation increases risk of neonatal mortality Pediatrics 2006:117(3):e380-e386. https://doi.org/10.1542/peds.2005-1496

27. Smith D. Caesarean section rates in South Africa 'recklessly high', warn experts. The Guardian 2014. https://www.theguardian.com/world/2014/sep/24/caesarean-section-south-africa (accessed 30 January 2018)

28. Mulol H, Coutsoudis A. Limitations of maternal recall for measuring exclusive breastfeeding rate in South African mothers. Int Breastfeed J 2018;13:19. https://doi.org/10.1186/s13006-018-0159-8

29. Sankar MJ, Sinha B, Chowdhury R, et al. Optimal breastfeeding practices and infant and child mortality: A systematic review and meta-analysis. Acta Paediatr 2015;104(S467):3-13. https://doi. org/10.1111/apa.1314

Accepted 12 June 2018. 